\title{
(Fehl-)Steuerung im Gesundheitswesen
}

\author{
Friedrich Overkamp ${ }^{a} \quad$ Klaus-Peter Thiele ${ }^{b} \quad$ Martin Wilhelm ${ }^{c}$ \\ a Praxis für Internistische Onkologie und Hämatologie, Recklinghausen, \\ ${ }^{\mathrm{b}}$ Kompetenz Centrum Onkologie der Medizinischen Dienste, Düsseldorf, \\ ${ }^{c}$ Medizinische Klinik 5, Schwerpunkt Onkologie/Hämatologie, Klinikum Nürnberg, Deutschland
}

\section{(Fehl-)Steuerung im ambulanten Bereich \\ Verantwortlicher Autor: Friedrich Overkamp, Recklinghausen}

Der Eid des Hippokrates («Ich werde ärztliche Verordnungen treffen zum Nutzen der Kranken nach meiner Fähigkeit und meinem Urteil, hüten aber werde ich mich davor, sie zum Schaden und in unrechter Weise anzuwenden ...») stellt das Patientenwohl in den Mittelpunkt ärztlichen Handelns. Es gilt das Primat «Primum non nocere», also in erster Linie Schaden vom Patienten abzuwenden. Was bedeutet dieser Eid 2500 Jahre später unter dem Eindruck einer zunehmenden Ökonomisierung der Onkologie, speziell im Hinblick auf unser Berufsethos?

Eine Ökonomisierung ist per se nicht verwerflich, zumal wirtschaftliches Handeln ein Gebot auch für Arztpraxen und Klinikambulanzen ist. Es besteht die Verpflichtung, wirtschaftlich angemessen und umsichtig zu verordnen. Ebenso ist es unstrittig, dass unternehmerische Aspekte inzwischen eine große Rolle spielen müssen bei der Organisation und Kostenkontrolle einer onkologischen Praxis/Tagesklinik, in Personalfragen und bei Entscheidungen für oder gegen bestimmte Kooperationen und Vernetzungen. Jedoch gibt es einige Aspekte ökonomischer Fehlsteuerungen, die zur Sorge Anlass geben.

\section{Ärztliches Gespräch und Betreuung grotesk unterbewertet}

Die Tätigkeit des Hämatologen und Onkologen ist sehr zeitaufwendig und überdurchschnittlich kommunikationsintensiv. Leider wird das ärztliche Gespräch weitgehend in Form einer Pauschale entgolten und ist damit eklatant unterbewertet. Das hat zur Konsequenz, dass nicht jeder Patient bei jedem Praxis- oder Ambulanzbesuch auch mit einem Arzt spricht - obwohl das in allen Belangen, die die Therapie be- treffen, zweifellos so sein sollte. Ein Mangel an Arzt-Patienten-Kontakten im Bereich der Onkologie birgt die Gefahr einer suboptimalen Betreuung und einer unzureichenden Supportivtherapie. Auch wenn ein Teil der Aufgaben von gut geschulten nichtärztlichen Mitarbeitern übernommen werden kann, bleibt das Arztgespräch ein zentraler Bestandteil der Patientenversorgung.

\section{Intravenöse Therapien werden besser vergütet als} subkutane

Auch im Hinblick auf die Vergütung der verschiedenen Applikationsformen onkologischer Therapien gibt es derzeit eine Fehlentwicklung. So werden intravenös zu applizierende Therapien deutlich besser vergütet als orale und subkutane Therapieformen (Abb. 1). Die nicht intravenösen Therapien sind jedoch alles andere als «einfach» und der damit verbundene Aufwand ist mindestens ebenso hoch wie bei intravenösen Therapien. Die noch relativ neuen, sogenannten zielge-

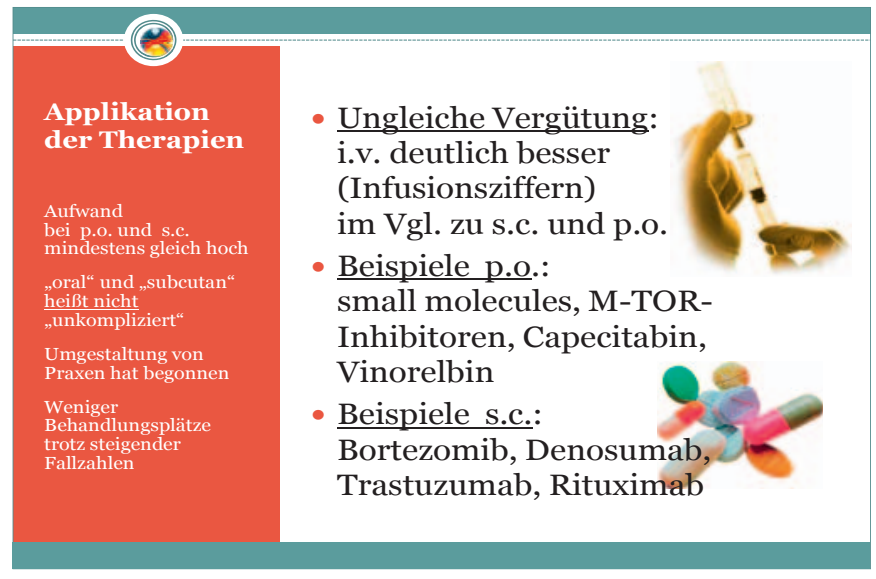

Abb. 1. Ungleiche Vergütung von intravenösen, subkutanen und oralen Therapien.

\begin{tabular}{ll}
\hline KARGER & @ 2013 S. Karger GmbH, Freiburg \\
0378-584X/13/3617-0020\$38.00/0 \\
$\begin{array}{l}\text { Fax +49 761 452 07 14 } \\
\text { Information@Karger.com }\end{array}$ & $\begin{array}{l}\text { Accessible online at: } \\
\text { www.karger.com }\end{array}$ \\
www.karger.com/onk
\end{tabular}


richteten Therapien (z.B. die orale Therapie mit Tyrosinkinasehemmern) haben ein anderes Nebenwirkungsprofil als konventionelle Chemotherapien und verlangen ein individuelles, auf die vom Patienten berichteten Beschwerden abgestimmtes Management der unerwünschten Wirkungen.

Die meisten der etwa 400 Substanzen in den Entwicklungspipelines der Pharmaindustrie sind orale Formulierungen, ein weiterer, ebenfalls relevanter Anteil ist subkutan zu applizieren.

In jüngster Zeit wurde erstmals ein monoklonaler Antikörper a priori in einer subkutanen Formulierung zugelassen (Denosumab). Andere, bereits seit langem intravenös verfügbare Antikörper werden künftig ebenfalls für die subkutane Injektion zur Verfügung stehen (z.B. Trastuzumab und Rituximab).

Zur Frage, ob und, wenn ja, wie stark sich die inadäquate Vergütung auf die onkologische Versorgung auswirkt, lassen sich derzeit nur Vermutungen anstellen. Es gibt jedoch aufgrund von Marktanalysen und Registeruntersuchungen Anlass zur Diskussion. So wissen wir aus dem Register der nichtkleinzelligen Lungenkarzinome (NSCLC), dass zwar über $60 \%$ der niedergelassenen Hämatologen/Onkologen bei Patienten mit NSCLC den Mutationsstatus des epidermalen Wachstumsfaktorrezeptors (EGFR) bestimmen (dies ist ein im Vergleich zu Kliniken erfreulich hoher Wert), dass jedoch etwa $30 \%$ der Patienten, für die entsprechend dem Testergebnis eine Erstlinientherapie mit einem Tyrosinkinasehemmer indiziert wäre, diesen nicht bekommen. Es ist unklar, womit dies zusammenhängt. Ein Grund ist sicher, dass viele NSCLCPatienten erst für die Zweit- oder Drittlinientherapie in die Praxen kommen und in der Erstlinientherapie bereits stationär eine Chemotherapie bekommen haben, ohne dass überhaupt eine EGFR-Mutationsanalyse durchgeführt wurde.

In den Kliniken spielen sicher die Kosten eine entscheidende Rolle, da der «Gentest» und die neuen zielgerichteten Therapien durchweg teurer sind als etablierte Standardchemotherapien und unter stationären Bedingungen kaum abzubilden sind. Bei den niedergelassenen Ärzten spielen aber auch ökonomische Überlegungen im Hinblick auf die Abrechenbarkeit eine Rolle bei der Auswahl der Therapie. Überspitzt könnte man sagen: In den Kliniken wird kaum getestet und aus Kostengründen primär chemotherapiert, in den Praxen wird großzügig getestet, aber zu spät, da die Patienten meist erst in fortgeschrittenen Phasen in die Praxen kommen. Und bei positiver Mutationsanalyse erhalten trotzdem nicht alle Patienten einen Tyrosinkinaseinhibitor.

Ein anderes Beispiel betrifft die antiresorptive Substanz Denosumab: Marktanalysen legen offen, dass subkutanes Denosumab von Klinikambulanzen und Organonkologen deutlich häufiger verordnet wird als von niedergelassenen Hämatologen/Onkologen. Dies hat ganz sicher etwas mit der Abrechenbarkeit im Vergleich zu intravenös applizierbaren Bisphosphonaten zu tun - und dies ist im Hinblick auf die bei Hämatologen und Onkologen viel höheren Fallzahlen auch verständlich.

\section{Arzneimittelkosten und Regressangst}

Ein schon seit langem bekanntes Beispiel aus der Gynäkologischen Onkologie betrifft die Aromataseinhibitoren: Solange sie patentgeschützt waren, wurden sie nicht so häufig verordnet, wie die Erkrankungszahlen es erwarten ließen. Inwieweit dies mit der Angst der Ärzte vor Regressen zusammenhing, sei dahingestellt. Das Verordnungsverhalten hat sich jedoch geändert, seit diese Substanzen generisch sind. Regressangst - ob begründet oder unbegründet - führt jedenfalls auch zu einer ökonomischen Fehlsteuerung.

Auch im Bereich der Hämatologie und Onkologie gab und gibt es in Marktanalysen auffallende Diskrepanzen zwischen Bedarfs- und Verordnungszahlen, hier z.B. im Hinblick auf den Einsatz von Tyrosinkinaseinhibitoren zur Behandlung von Patienten mit chronischer myeloischer Leukämie (CML).

\section{Quartalsabrechnungen der Kassenärztlichen Vereinigungen:} ungerecht und unausgewogen

Das gesamte derzeitige Abrechnungssystem kann aus der Sicht eines niedergelassenen Onkologen ohne Übertreibung als eine weitgehende ökonomische Fehlsteuerung bezeichnet werden. Auf die inadäquate Vergütung des ärztlichen Gespräches, die fehlende Vergütung oraler und subkutaner Therapien wurde bereits hingewiesen. Aber auch die Modalitäten der Quartalsabrechnungen selbst erscheinen ungerecht (was z.B. die Verteilung der Regelleistungsvolumina (RLV) und der qualitätsbezogenen Zusatzvolumina (QZV) angeht) oder gar absurd (z.B. was die teilweise gravierend unterschiedliche Vergütung exakt gleicher Leistungen in den verschiedenen Kassenärztlichen Vereinigungen (KV) betrifft). Im Bereich der KV Westfalen-Lippe beispielsweise werden mehr als ein Drittel aller in einem Quartal erbrachten Leistungen gar nicht vergütet. Niemand wird ernsthaft nur zwei Monate eines Quartals seine Praxis zur Therapie öffnen und im dritten Monat geschlossen halten, nur weil der dritte Monat ohne Leistungsvergütung bestritten werden muss. Aber auch wenn

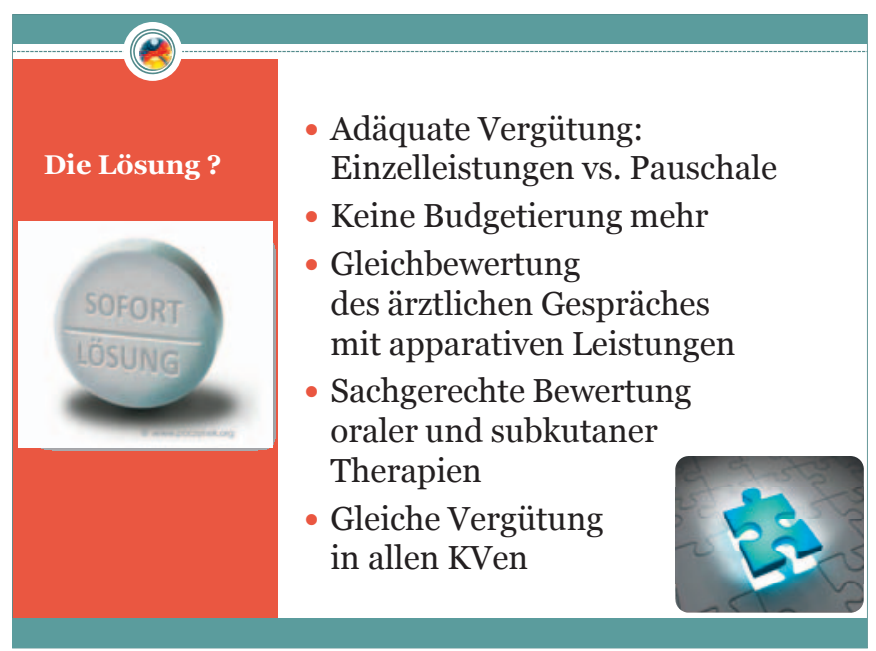

Abb. 2. Lösungsansätze für eine bessere Verteilung der Ressourcen. 
es unbequem ist, dies auszusprechen: Die ökonomische Situation hat eben doch Auswirkungen auf die unternehmerischen Entscheidungen der Ärzte bezüglich der Praxisschwerpunkte. Folgende Optionen finden beispielsweise Berücksichtigung (ohne Bewertung): Beteiligung an der Nachsorge, Beteiligung an klinischer Forschung und Versorgungsforschung, Implementierung von individuellen Gesundheitsleistungen (IGeL), Nutzung von Zuweiserpauschalen.

Manche dieser Optionen sind von sehr hohem Wert, aber ökonomisch auch problematisch (z.B. klinische Studien), andere von zweifelhaftem Wert, aber ökonomisch hilfreich (z.B. Zuweiserpauschalen oder IGeL).

\section{Lösungsansätze}

Um die skizzierten ökonomischen Missstände besser zu steuern, werden verschiedene Lösungen diskutiert (Abb. 2). Mit der Umsetzung dieser wenigen Maßnahmen wäre bereits viel gewonnnen.

\section{(Fehl-)Steuerung in der Klinik}

\section{Verantwortlicher Autor: Martin Wilhelm, Nürnberg}

In Zusammenhang mit den steigenden Kosten im Gesundheitswesen sehen sich die Kliniken häufig dem Vorwurf ausgesetzt, als wesentlicher Kostenfaktor immer noch nicht alle Effizienzreserven erschlossen zu haben und sich stattdessen auf Fallzahlsteigerung und gewinnbringende invasive Eingriffe zu konzentrieren. So äußerte sich Bundesgesundheitsminister Daniel Bahr beim 35. Deutschen Krankenhaustag im Jahr 2012: «Tatsache ist, dass die Krankenhausausgaben jährlich um $4 \%$ steigen, das ist mehr als in allen anderen Bereichen der GKV». Bei näherer Betrachtung der Ausgaben für Krankenhausbehandlungen, Arzneimittel und ambulante Behandlungen und deren Veränderung in den letzten 5 Jahren wird jedoch deutlich, dass die Kliniken keinen überproportional hohen Anteil an der Kostensteigerung haben, da die Kosten für Arzneimittel bei ambulanten Behandlungen als zusätzlicher Ausgabenposten hinzuzurechnen sind. Die Steigerung für die stationären Ausgaben (von 2006 bis 2011 um etwa $20 \%$ ) war damit vergleichbar mit der Steigerung der Gesamtausgaben im ambulanten Bereich von etwa $22 \%$ im gleichen Zeitraum.

In einem Schreiben vom Bundestagsabgeordneten Jens Spahn an die Mitglieder der CDU/CSU-Fraktion vom 9. Januar 2013 heißt es: «... hat sich die Gesamtlage der Krankenhäuser durchaus positiv entwickelt. Nach dem DKIKrankenhaus Barometer [DKI = Deutsches Krankenhausinstitut] haben deutschlandweit im Jahr 2002 noch über 40\% einen Fehlbetrag verbucht, bis 2010 hat sich der Anteil auf $21 \%$ halbiert.» Nicht erwähnt wurde, dass seit dem Jahr 2000 etwa 200 Krankenhäuser (etwa 10\%) schließen mussten und sich folglich allein dadurch der prozentuale Anteil der nicht defizitär arbeitenden Häuser verringerte. Betrachtet man zudem aktuellere Statistiken, hat der Anteil der defizitär arbeitenden Krankenhäuser einen Anteil von 47\% (Stand 2012; Bayerische Krankenhausgesellschaft) bzw. 46-54\% (Verband der Krankenhausdirektoren) an der Gesamtzahl der Kliniken.

Auch bei der Interpretation der Ursachen für die $\mathrm{Zu}$ nahme invasiver Eingriffe würde man sich eine objektivere Beurteilung wünschen. Allgemein wird die Auffassung vertreten, dass die Leistungsausweitung nur teilweise auf die Alterung der Bevölkerung und die damit einhergehende steigende Morbidität zurückzuführen ist und zu einem beträchtlichen Teil durch die Gewinnbestrebungen der Akteure zu erklären ist. Die Kritik bezieht sich z.B. auf die deutliche $\mathrm{Zu}-$ nahme bei der Implantation von Hüft- und Kniegelenkprothesen innerhalb der letzten Jahre. Dieser Anstieg erklärt sich jedoch weitgehend durch vermehrte Revisions- und Wechseloperationen, während dagegen die Zahl der Erstimplantationen stagniert oder zurückgeht! Daher kann man daraus keinen Hinweis für einen «systematischen Fehlanreiz», der zu einer ökonomisch begründeten Fallzahlausweitung geführt hätte, ableiten, zumal alle Eingriffe der externen stationären Qualitätssicherung unterliegen [1]. Jede Fallzahlsteigerung als primär ökonomisch bedingt $\mathrm{zu}$ interpretieren, ist einer objektiven Diskussion nicht dienlich. Vielmehr sollte man überlegen ob nicht auch die Ansprüche der Patienten mit dem berechtigten Wunsch eines körperlich aktiven Lebens bis ins hohe Alter für die Steigerung der Eingriffe verantwortlich sein könnten. Vonseiten der politisch Verantwortlichen wird man nicht müde zu betonen, dass im deutschen Gesundheitssystem «alle Leistungen für alle Patienten zur Verfügung stehen». Ein weiterer Vorwurf lautet, die Krankenhäuser würden sich zunehmend allein über die Mengenausweitung ihrer Leistungen refinanzieren. Dies ist zumindest für solche Krankenhäuser schwer vorstellbar, deren Patienten weitgehend über Notfallaufnahmen und durch niedergelassene Ärzte eingewiesen werden. Da nicht a priori vereinbarte Mehrleistungen zudem mit kräftigen Abschlägen (bis zu 65\%) und damit im Wesentlichen nur die anfallenden Sachleistungen vergütet werden, ist eine solche Strategie mittelfristig zum Scheitern verurteilt, da sie ja auch nur funktionieren kann, wenn die zusätzlich behandelten Patienten nicht durch eine Zunahme der Arzt- bzw. Pflegestellen ausgeglichen werden. Diskussionen auf Basis einseitiger Interpretationen von Statistiken sind wenig hilfreich und dienen eher dazu, von den eigentlichen Problemen abzulenken, anstatt gemeinsam nach Lösungen zu suchen.

\section{Medizinischer Fortschritt als Kostentreiber}

Die steigenden Kosen im Gesundheitswesen sind aktuell und in Zukunft in noch größerem Ausmaß auf den medizinischen Fortschritt zurückzuführen. Das betrifft auch die onkologischen Therapien. Zum Beispiel wurden Patienten mit Multiplem Myelom bis vor einigen Jahren standardmäßig mit 
der Kombination Melphalan/Prednison behandelt, wofür pro Jahr etwa 120 Euro zu veranschlagen waren. Für die heutige Therapie mit Bortezomib oder Lenalidomid muss dagegen mit Jahrestherapiekosen von etwa 50000 Euro pro Patient kalkuliert werden. Kombinationstherapien sind entsprechend noch teurer.

Neue Therapieverfahren sind häufig nicht nur effektiver, sondern auch verträglicher, sodass damit auch ältere und komorbide Patienten behandelt werden können. Laut Prognose des Deutschen Instituts für Wirtschaftsforschung wird der Beitragssatz in der Gesetzlichen Krankenversicherung (GKV) bis zum Jahr 2040 auf 23\% steigen, wenn man nur den medizinisch-technischen Fortschritt vollumfänglich berücksichtigt.

\section{Ökonomisierung der Medizin}

Um der Kostensteigerung bzw. dem Einnahmerückgang im Gesundheitswesen zu begegnen, wurden seit 1977 über 20 Gesetze verabschiedet. Dabei handelte es sich im Wesentlichen um Kostendämpfungsgesetze, die die Regulierungsdichte erhöht und den ökonomischen Druck auf die Kliniken wesentlich erhöht haben, ein langfristig stabilisierender Effekt ist aber ausgeblieben. Obwohl die Einnahmen der Krankenhäuser zunahmen, sind die Ausgaben für Personal, Medikamente/Verbrauchsmittel oder Energiekosten überproportional gestiegen. Allein die Schere zwischen Lohnkostensteigerung und den Einnahmen der Krankenhäuser geht immer weiter auseinander. Um dem gegenzusteuern, wurden defizitäre Krankenhäuser geschlossen und in den anderen mehr Patienten in kürzerer Zeit versorgt. So sind in den Jahren 2000 2011 die Fallzahlen um 6\% gestiegen (von 17,3 auf 18,3 Millionen), die stationäre Verweildauer der Patienten hat sich um $21 \%$ verkürzt (von 9,7 auf 7,7 Tage) und die Zahl der Krankenhausbetten hat um 10\% abgenommen (Quelle: destatis). Ein Vergleich der Leistungszahlen der in der Arbeitsgemeinschaft der Hämatologen und Onkologen im Krankenhaus e.V. (ADHOK) zusammengeschlossenen Krankenhäuser weist allein für den relativ kurzen Zeitraum 2009-2011 eine erhebliche Steigerung bei den Kennzahlen Fälle/Arzt oder Casemix (CM)/Arzt auf (Tab. 1). Der ökonomische Druck führte somit zu einer immer weiter zunehmenden Arbeitsbelastung des einzelnen Mitarbeiters: Jedem muss klar sein, dass dies nicht beliebig fortgesetzt werden kann.

Die überwiegende Mehrzahl der Kliniken ist der Ansicht, dass die Zeiten, in denen die Krankenhäuser über eine größere Effizienzreserve verfügten, vorbei sind. Je mehr die Reserven abnehmen, umso größer wird aber unweigerlich der ökonomische Druck durch die Kostensteigerungen. Dies führt bereits jetzt dazu, dass die Ökonomie die Medizin in Teilen bestimmt. Zielvereinbarungen in Form von Boni sind nichts anderes als der Versuch, Einfluss auf die medizinischen Entscheidungen der Ärzte zu nehmen. Dass es dadurch bewusst oder unbewusst zu medizinisch fragwürdigen Entscheidungen für Eingriffe kommt, die für das Krankenhaus besonders lukrativ sind, ist nicht verwunderlich. Die Klinikmitar-
Tab. 1. Benchmarking: Leistungszahlen ADHOK-Krankenhäuser im Vergleich der Jahre 2009 und 2011 (Quelle: ADHOK)

\begin{tabular}{llll}
\hline & 2009 & 2011 & Differenz \\
\hline Fälle/Arzt & 222 & 246 & $+11 \%$ \\
CM/Arzt & 234 & 265 & $+13 \%$ \\
Arztkosten/CM & $474 €$ & $506 €$ & $+7 \%$ \\
Ärzte/Bett & 0,177 & 0,171 & $-3 \%$ \\
\hline
\end{tabular}

beiter sind mit einem Anteil von $60-70 \%$ der wesentliche Kostenfaktor eines Krankenhauses. Die Kalkulation der Stellen auf eine Mindestbesetzung führt unweigerlich zu häufigen und lang andauernden Überlastungen in Phasen der Volloder Überbelegung. Dies hat erhebliche Konsequenzen nicht nur für die Mitarbeiterzufriedenheit, sondern auch für die Qualität der Arbeit. So konnte eindeutig gezeigt werden, dass ein «Overcrowding» der größte Risikofaktor für nosokomiale Infektionen in Krankenhäuser ist [2]. Daran wird auch die zwangsweise Einführung zusätzlicher aus DRG-Erlösen (DRG = diagnosebezogene Fallgruppen) zu finanzierender hygienebeauftragter Ärzte oder weiterer Qualitätsmanagement (QM)-Maßnahmen wenig ändern.

\section{Drohende Entwertung der Berufe im Gesundheitswesen}

Der von der weitreichenden Ökonomisierung ausgehende Druck führt zu einer Entwertung der Arzt- und Pflegeberufe [3]. Nur solche Kliniken werden Bestand haben, die möglichst viele Patienten in möglichst kurzer Zeit mit dem geringsten Versorgungsaufwand durchschleusen. Die «sprechende Medizin» ist in diesem System ein Anachronismus; sie ist zeitaufwendig und deshalb hinderlich. Der ärztliche Blick verengt sich auf DRG-fähige Hauptdiagnosen und die fachlichen Ermessensspielräume werden eingeschränkt. Alle Abläufe müssen permanent kontrolliert und dokumentiert werden; was nicht funktioniert, wird sanktioniert [3].

Mit der Einführung von DRG auch in der Palliativmedizin wird die Ökonomisierung auch dort zum bestimmenden Faktor. Patienten nach Diagnosen zu verschlüsseln, obwohl die in der Palliativmedizin von nachrangiger Bedeutung sind, ist ebenso inakzeptabel wie die Katalogisierung dieser Patienten anhand von Fallpauschalen und mittlerer Verweildauer.

Unter solchen Bedingungen wird sich ein vertrauensvolles Arzt-Patienten-Verhältnis nur schwer verwirklichen lassen. In der logischen Konsequenz dieses Denkens wird der Patient zum Kunden, wodurch sich in Verbindung mit der ständigen Zeitknappheit unweigerlich eine emotionale Distanz zwischen Arzt und Patient entwickelt [3].

Nur so ist zu erklären, dass eine implizite (versteckte) Rationierung in einem erschreckenden Umfang bereits in der Medizin stattfindet, wie Beispiele aus der Kardiologie und Intensivmedizin zeigen (ähnliche Situationen gibt es aber laut einer Umfrage der Deutschen Gesellschaft für Hämatologie und Medizinische Onkologie (DGHO) auch in der Onkologie) [4]. 
Abb. 3. Hindernisse auf dem Weg zu einem angemessenen Preis/Leistungs-Verhältnis.

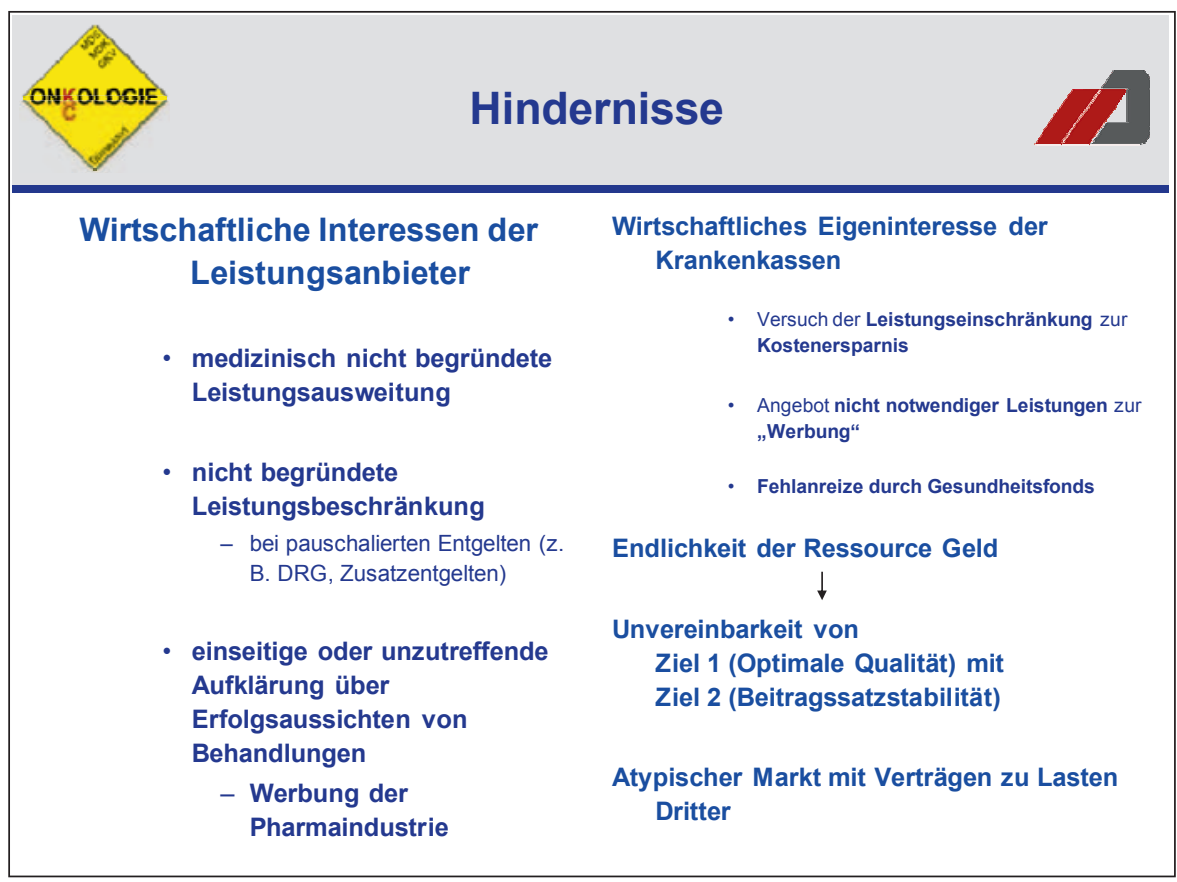

Offenbar wird bereits jetzt schon immer wieder am Krankenbett nach persönlichem Ermessens des Arztes nach dem QALY-Konzept (QALY = qualitätskorrigiertes Lebensjahr) gearbeitet.

Meiner Ansicht nach besteht das wesentliche Versäumnis der Entscheidungsträger darin, sich in ihrem Maßnahmen allein darauf zu konzentrieren, Kostendämpfungsgesetze zu erlassen und damit den ökonomischen Druck auf die Kliniken so stark zu erhöhen, dass die Medizin sich in einem Ausmaß verändert, wie weder die Behandler noch die Betroffenen es wollen. Ich würde mir eine offene Diskussion wünschen, in der mit Hinweis auf eine durch den medizinischen Fortschritt in Zukunft fehlende Finanzierbarkeit unseres Gesundheitssystems zumindest die beiden Auswege artikuliert werden, ob künftig deutlich mehr Geld in das Gesundheitswesen fließen soll oder aber ob klare Regelungen für eine explizite (offene) Rationierung zu treffen sind.

\section{(Fehl-)Steuerung aus Sicht der Medizinischen Dienste}

\section{Verantwortlicher Autor: Klaus-Peter Thiele, Düsseldorf}

Die Festlegung von Kriterien für eine gerechte Ressourcenverteilung im Gesundheitswesen ist eine politische Aufgabe, wie der Deutsche Ethikrat im Jahr 2011 betont hat. Die Thematik berühre medizinische, ökonomische, ethische und juristische Fragestellungen, wobei aufgrund ihrer Komplexität ein vollständiger Konsens zwischen allen Beteiligten unmöglich sei. Allerdings führt der gezieltere Einsatz von Geldern nicht automatisch zu einer besseren Versorgung der Pa- tienten. Schon jetzt werden die Versorgungsangebote nicht umfassend genutzt, wie einige vom Bundesministerium für Gesundheit (BMG) geförderte Feldstudien in Bayern belegen. Demnach erhalten zwischen 10 und $60 \%$ der Patientinnen mit lokalisiertem Brustkrebs nicht die dem wissenschaftlichen Standard entsprechende adjuvante Chemotherapie [5]. Auch werden mehr als 50\% aller Patienten mit Rektumkarzinom im Stadium II/III nicht gemäß Standard (neo)adjuvant mit Radiochemotherapie behandelt [6]. Dass so jährlich mehrere Tausend Patienten in Deutschland vermeidbare Tumorrezidive erleiden, ist ein weiteres Indiz für die mangelhafte Ausschöpfung der vorhandenen Ressourcen.

Die in den gesetzlichen Krankenkassen versicherten Patienten dürfen nach derzeitiger Rechtslage die bestmögliche Qualität der medizinischen Versorgung erwarten, was im Übrigen auch den Schutz vor nicht notwendigen Therapien einschließt. Sie haben Anspruch auf gleichförmige Versorgung über Kassen- und Ländergrenzen hinweg [7]. Da aber andererseits auch die Stabilität der Beitragssätze im Interesse der Versicherten ist, muss die medizinische Qualität zu einem wirtschaftlich angemessenen Preis angeboten werden. Dies wird behindert durch finanzielle Interessen sowohl der Leistungsanbieter als auch der Krankenkassen sowie durch die Begrenztheit der Mittel (Abb. 3).

In diesem Zusammenhang ist auch das Hinzuziehen von Studien, insbesondere von Zulassungsstudien, zur Festlegung von Therapiekonzepten kritisch zu hinterfragen. Zulassungsstudien sind für Pharmahersteller von großer wirtschaftlicher Bedeutung, weshalb häufig ein Studiendesign gewählt wird, das ein für das Prüfmedikament positives Studienergebnis erwarten lässt. Dies kann etwa dadurch erreicht werden, dass die Studienteilnehmer eine Reihe von «idealen» Einschluss- 
Abb. 4. Bedeutung der AMNOG-Bewertung für die Versorgung.

- Instrument zur Preisregulierung, bindet Medikamentenpreis an den Zusatznutzen.

- „Gerechte" Profite für Hersteller, Anreiz zur Entwicklung neuer Medikamente mit besonders günstigen Behandlungsergebnissen.

Hoffnung: Verbesserung der Ressourcenallokation und Versorgungsoptimierung.

- Umfassende Bewertung von Nutzen und Zusatznutzen eines Medikamentes für die Gesamtpopulation der Patienten auf Basis von Zulassungsstudie und AMNOG-Bewertung („in der Regel zu frühe Nutzenbewertung“) in aller Regel nicht möglich.

- Zur Nutzenbewertung in klinisch relevanten Untergruppen und zur Optimierung der Therapiestrategie sind weitere klinische Studien nach der Zulassung zwingend notwendig.

- Wie wären Imatinib, Trastuzumab, Rituximab bewertet worden ...?

kriterien aufweisen müssen, wobei solche Patienten im klinischen Alltag eher selten sind. Oder die Effektivität der Therapie wird anhand weicher Endpunkte (Time-to-Progression, Clinical Benefit) gemessen bzw. es wird eine absehbar weniger wirksame Vergleichstherapie gewählt. Es ist im Interesse der Firmen, solche Studien möglichst kostengünstig durchzuführen und dabei mit relativ wenigen Patienten zu schnellen Ergebnissen zu kommen.

Hilfreicher aus Sicht der Arnzeimittelbewerter sind versorgungsorientierte Studien, die eine Optimierung einer Therapiestrategie (und nicht einer Unternehmensstrategie!) zum Ziel haben. Eine solche Studie hat bezüglich ihres Aufbaus eine möglichst große Relevanz für die Versorgung, sie bildet ein repräsentatives Patientenkollektiv ab, verwendet patientenrelevante Studienendpunkte (Gesamtüberleben, Lebensqualität) und verfügt über einen evidenzbasierten Vergleichsarm. Versorgungsorientierte Studien umfassen naturgemäß viele Patienten und haben eine lange Nachbeobachtungszeit.

\section{AMNOG-Bewertung und Versorgungsrealität}

Für die Nutzenbewertung neuer Medikamente nach dem Arzneimittelmarktneuordnungsgesetz (AMNOG) können zwangsläufig nur die zur Zulassung bereits vorliegenden Daten herangezogen werden, was aus den genannten Gründen eine faire Bewertung schwierig macht. Auch die auf Basis der AMNOG-Bewertung erfolgte Nutzenbeurteilung durch den Gemeinsamen Bundesausschuss (G-BA) wirft Fragen auf: So wurde dem Wirkstoff Abirateron $\left(\right.$ Zytiga $^{\circledR}$ ) ein «beträchtlicher» Zusatznutzen für die Behandlung bestimmter Patienten mit metastasiertem Prostatakarzinom bescheinigt; dem Wirkstoff Cabazitaxel (Jevtana ${ }^{\circledR}$ ) wurde für die gleiche Indikation ebenfalls das Vorliegen eines - allerdings geringen
- Zusatznutzens eingeräumt. Lässt sich daraus für die allgemeine Versorgung dieser Patienten ableiten, dass Abirateron grundsätzlich gegenüber Cabazitaxel bevorzugt werden sollte? Mangels direkter Vergleichsstudien ist eine solche Schlussfolgerung für die Versorgung nicht möglich. Insbesondere ist nicht auszuschließen, dass ein unterschiedliches Vorgehen sinnvoll wäre, z.B. die Anwendung von Abirateron für ältere Patienten (wegen der wahrscheinlich geringeren Toxizität der Substanz) und die Behandlung mit Cabazitaxel für jüngere Patienten mit rasch progredientem Tumor. Für beide Präparate könnte letztlich die Nutzen-Risiko-Abwägung ungünstig ausfallen, da in den Studien (im Gegensatz zur Versorgungsrealität) nur wenige ältere Patienten mit Begleiterkrankungen vertreten waren.

Außer zur Nutzenbewertung gibt es zu Abirateron und Cabazitaxel noch weitere, die Patientenversorgung betreffende Fragen, die mit der Zulassung durch die European Medicines Agency (EMA) allein nicht zu beantworten sind. Da die Entscheidungen der EMA nicht immer nachvollziehbar sind (das betrifft z.B. die Zulassung von Lapatinib), ist die Arbeit nationaler Bewertungsstellen wie das Institut für Qualität und Wirtschaftlichkeit im Gesundheitswesen (IQWiG) aus Sicht der Medizinischen Dienste im Gesundheitswesen sehr hilfreich. Im Wesentlichen handelt es sich bei der Bewertung nach AMNOG allerdings um ein Instrument der Preisgestaltung, das als Notbremse gegen inflationäre Anstiege bei den Herstellerpreisen fungieren soll (Abb. 4).

Angesichts der Defizite der AMNOG-Bewertung in Fragen der Patientenversorgung sollten im Kontext mit der Versorgungsforschung andere Wege beschritten werden, um die Anwendung neuer Medikamente (oder auch Behandlungsmethoden) zu Lasten der GKV zu regeln. So wären nach der Zu- 
Abb. 5. Versorgungsforschung zur Nutzenbewertung.

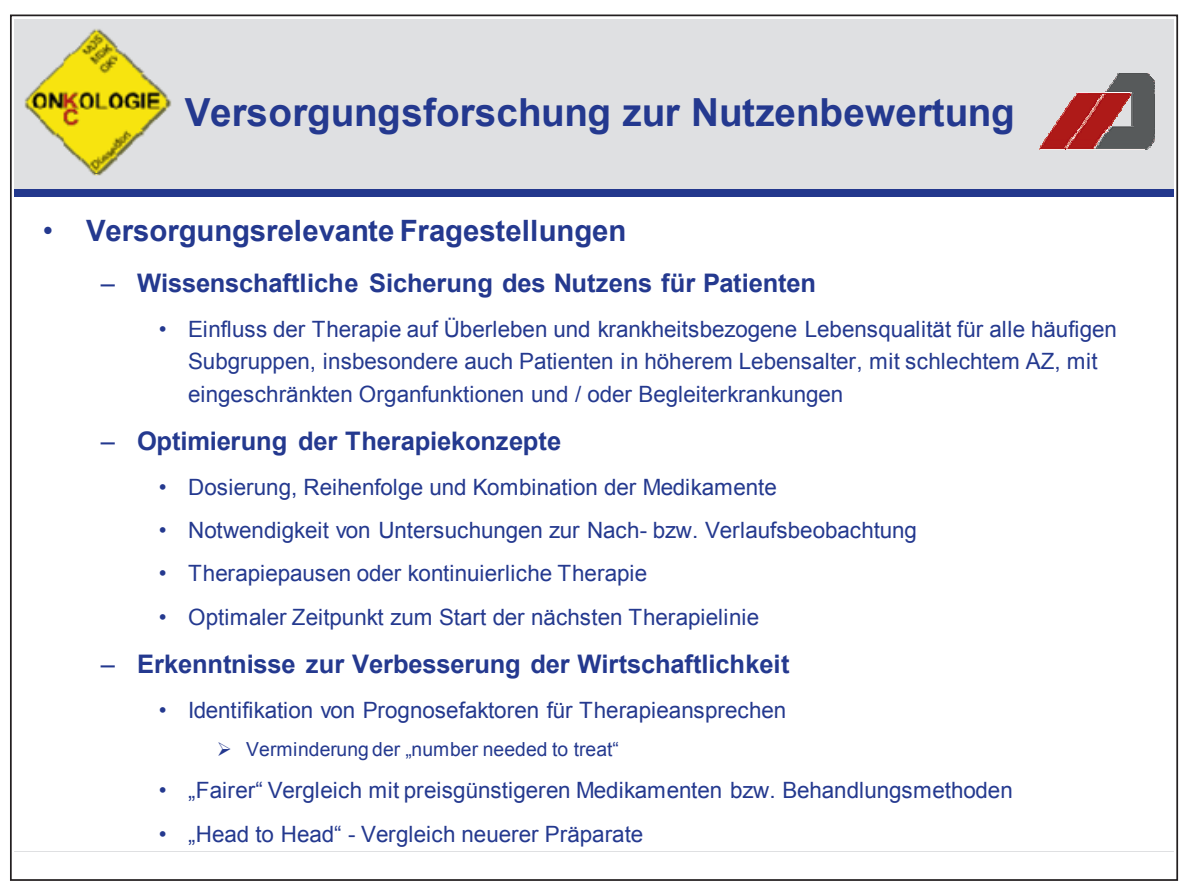

lassung eines Arzneimittels weitere Studien in Kooperation von GKV und Studiengruppen nötig. Die Anwendung eines neuen Präparates sollte zunächst auf angeschlossene Prüfzentren beschränkt sein und die Aufnahme des Patienten in Studienprotokolle vorsehen.

Davon auszunehmen sind Heilversuche nach Kriterien des Bundesverfassungsgerichtes, für die jedoch ebenfalls eine Behandlung in Prüfzentren mit Erfassung und Auswertung der Daten zu fordern ist. Erst nach Klärung der versorgungsrelevanten Fragen sollte das Medikament allgemein verordnungsfähig sein. Besonders wichtig für die Abschätzung des Nutzens sind frühzeitige Head-to-Head-Vergleiche, wie sie auf Initiative der pharmazeutischen Industrie bisher nur selten vorgenommen werden.

Um die skizzierten neuen Konzepte umzusetzen, müssen die gesetzlichen Rahmenbedingungen weiterentwickelt werden, das tangiert insbesondere die Anforderungen an die «Erprobung von Untersuchungs- und Behandlungsmethoden» (§ 137e SGB V) und an die «Ambulante spezialfachärztliche Versorgung» ( $\$ 116$ b SGB V). Zu hinterfragen ist auch die Regelung, wonach Orphan Drugs bereits mit der Zulassung ihren Zusatznutzen hinlänglich nachgewiesen haben. Da mit Ausnahme der Karzinome in Brustdrüse, Lunge, Kolorektum und Prostata alle Tumore als selten eingeordnet werden, entgehen viele onkologische Präparate einer frühen Nutzenbewertung aufgrund ihres Orphan-Drug-Status. Pro Jahr wird in Deutschland deutlich über eine Milliarde Euro Umsatz mit zugelassenen Orphan Drugs erzielt. Zwar müssen sich Orphan Drugs, deren Jahresumsatz 50 Millionen Euro überschreitet, einer nachträglichen Nutzenbewertung nach
AMNOG unterziehen, doch achten die Anbieter dieser Produkte im Hinblick auf eine drohende Nutzenbewertung auf eine entsprechend geschickte Preisgestaltung.

Viele der offenen, für die Patientenversorgung relevanten Fragen zum Einsatz neuer Arzneimittel (Abb. 5) könnten durch eine Optimierung der Datenlage beantwortet werden. Wenn entsprechend sichere Daten zur Verfügung stehen, dürfte die Nutzenbewertung von Ärzten, Patienten und Öffentlichkeit anders wahrgenommen werden. Durch Verzicht auf Behandlungen ohne gesicherten Nutzen sollte es möglich sein, den hohen Qualitätsanspruch an onkologische Therapien aufrechtzuerhalten und zugleich Kosten einzusparen. Es erschlössen sich somit neue finanzielle Ressourcen, die z.B. für die angemessene Honorierung primär ärztlicher Leistungen wie Anamnese und körperliche Untersuchung, für die Beratung von Patienten und Angehörigen sowie für Hausbesuche genutzt werden könnten. Vielleicht wäre auch endlich genug Geld da für zusätzliche Angebote im onkologischen Bereich, etwa in der Palliativmedizin, der qualifizierten ambulanten Pflege oder auch der Psychotherapie und Seelsorge. Die Bereitstellung der dafür nötigen Mittel durch Einsparung an anderer Stelle wäre eine wichtige Maßnahme zur Korrektur von Fehlsteuerungen im Gesundheitswesen.

\section{Disclosure Statements}

F.O. und M.W. geben an, dass keine Interessenkonflikte bestehen. K.-P.T. ist Angestellter einer Einrichtung der Gesetzlichen Krankenversicherung und der Medizinischen Dienste der Gesetzlichen Krankenversicherung. 


\section{Literatur}

1 Franz D, Roeder N: Mengendynamik in den Krankenhäusern: auch eine gesellschaftliche Frage. Dtsch Arztebl 2012;109:A-2580/B-2116/C-2068.

-2 Schwab F et al.: Understaffing, overcrowding, inappropriate nurse:ventilated patient ratio and nosocomial infections: which parameter is the best reflection of deficits? J Hosp Infect 2012;80:133-139.

3 Maio G: Gesundheitswesen: ärztliche Hilfe als Geschäftsmodell? Dtsch Arztebl 2012;109:A-804/ B-696/C-692.
4 Strech D et al.: Ausmaß und Auswirkungen von Rationierung in deutschen Krankenhäusern - ärztliche Einschätzungen aus einer repräsentativen Umfrage. Dtsch Med Wochenschr 2009;134:1261-1266.

5 Engel J et al.: Qualitätssicherung in der Onkologie am Beispiel des Mammakarzinoms der Feldstudie München. Onkologe 2001;7:307-320.
6 Engel J et al.: Quality of treatment in routine care in a population sample of rectal cancer patients. Acta Oncol 2005;44:65-74.

7 Urteil des Bundesverfassungsgerichtes vom 17.12.2002, Az. 1 BvL 28/95, 1 BvL 29/95 und 1 BvL 30/95. 